\title{
Three-dimensional numerical simulations of equatorial spread $F$ : Results and observations in the Pacific sector
}

\author{
H. C. Aveiro, ${ }^{1}$ D. L. Hysell, ${ }^{1}$ R. G. Caton, ${ }^{2}$ K. M. Groves, ${ }^{3}$ J. Klenzing, ${ }^{4}$ R. F. Pfaff, ${ }^{4}$ \\ R. Stoneback, ${ }^{5}$ and R. A. Heelis ${ }^{5}$ \\ Received 13 August 2011; revised 30 January 2012; accepted 31 January 2012; published 21 March 2012.
}

[1] A three-dimensional numerical simulation of plasma density irregularities in the postsunset equatorial $F$ region ionosphere leading to equatorial spread $F$ (ESF) is described. The simulation evolves under realistic background conditions including bottomside plasma shear flow and vertical current. It also incorporates C/NOFS satellite data which partially specify the forcing. A combination of generalized Rayleigh-Taylor instability (GRT) and collisional shear instability (CSI) produces growing waveforms with key features that agree with C/NOFS satellite and ALTAIR radar observations in the Pacific sector, including features such as gross morphology and rates of development. The transient response of CSI is consistent with the observation of bottomside waves with wavelengths close to $30 \mathrm{~km}$, whereas the steady state behavior of the combined instability can account for the $100+\mathrm{km}$ wavelength waves that predominate in the $F$ region.

Citation: Aveiro, H. C., D. L. Hysell, R. G. Caton, K. M. Groves, J. Klenzing, R. F. Pfaff, R. Stoneback, and R. A. Heelis (2012), Three-dimensional numerical simulations of equatorial spread $F$ : Results and observations in the Pacific sector,

J. Geophys. Res., 117, A03325, doi:10.1029/2011JA017077.

\section{Introduction}

[2] Around sunset, the loss of dominance of the $E$ region dynamo to the $F$ region creates a shear in the horizontal plasma flow at the $F$ region bottomside [Haerendel et al., 1992]. Above the shear node, the vertical electric field points downward, and the plasma drifts eastward. Below the node, the vertical electric field points upward, and the plasma drifts westward, even though the neutral flow is everywhere eastward. The imperfectly efficient $F$ region dynamo sets up a vertical current supplied by field-aligned currents connecting the equatorial $F$ region to the $E$ layer at low latitudes to midlatitudes. Related to this phenomenon, a family of plasma irregularities collectively referred to as equatorial spread $F$ (ESF) disturbs the nighttime equatorial $F$ region (see Woodman [2009] for details). Those irregularities can be detected by incoherent and coherent scatter radars after sunset and appear in three different classes (or stages): topside, bottomside, and bottom-type.

[3] Topside irregularities are driven by the generalized Rayleigh-Taylor instability (GRT) near and above the $F$ peak. Typical GRT growth rates at the $F$ peak are $\sim 10^{-3} \mathrm{~s}^{-1}$

\footnotetext{
${ }^{1}$ Earth and Atmospheric Sciences, Cornell University, Ithaca, New York, USA.

${ }^{2}$ Space Vehicles Directorate, Air Force Research Laboratory, Kirtland AFB, New Mexico, USA.

${ }^{3}$ Space Vehicles Directorate, Air Force Research Laboratory, Hanscom AFB, Massachusetts, USA.

${ }^{4}$ NASA Goddard Space Flight Center, Greenbelt, Maryland, USA.

${ }^{5}$ Hanson Center for Space Sciences, University of Texas at Dallas, Richardson, Texas, USA.

Copyright 2012 by the American Geophysical Union 0148-0227/12/2011JA017077
}

[Sultan, 1996; Hysell et al., 2006], which implies no more than 3-4 e-folds per hour. This is a universal instability (i.e., nearly scale independent), and thus GRT theory cannot explain by itself the predominant scale sizes of ESF irregularities.

[4] At altitudes below the plasma shear node, the differential motion between plasma and neutrals and associated vertical current provide a source of free energy that can drive collisional shear instability (CSI). During the initial transient phase, the zonal wavelength of the fastest growing mode $(\lambda)$ is approximately twice the density gradient scale length $\left(L^{-1}=\frac{1}{n} \frac{\partial n}{\partial z}\right)$, i.e., $\lambda \simeq 2 L$. Longer wavelengths dominate during the asymptotic phase, with $\lambda \simeq 4 \pi L$ [Hysell and Kudeki, 2004]. For typical bottomside gradient scale lengths, the zonal wavelengths for the transient and asymptotic growth phase are expected to be $\sim 30 \mathrm{~km}$ and $100+\mathrm{km}$, respectively. The irregularities produced by CSI propagate obliquely at an intermediary angle between upward and westward. CSI growth rates are faster than that of GRT under nominal conditions. Typical growth rates are $\sim 5 \times$ $10^{-3} \mathrm{~s}^{-1}$, or 18 e-folds per hour during the transient phase [Kudeki et al., 2007]. CSI therefore can account for the scales and rapid growth of the dominant irregularities observed by radar in ESF. Bottom-type layers are almost ubiquitous over Jicamarca immediately after sunset, and their patchy signature seem to signal the onset of the CSI [Hysell et al., 2004]. Bottomside irregularities generated by CSI remain confined to the base of the $F$ region, however, and are therefore not space weather effective. In order to bridge the gap between irregularities at the bottomside and the topside, zonal electric fields are generally needed to propel irregularities toward the $F$ peak, leading to topside spread $F$. 
[5] In recent years, efforts to simulate ESF in three dimensions have been made [Huba et al., 2008; Retterer, 2010; Keskinen, 2010]. These simulations are driven in such a way as to emphasize the role of GRT while mainly neglecting CSI. More recent work has shown that the two instabilities function together in nature: CSI appears neither to suppress GRT nor merely to seed it; rather, the two mechanisms appear to grow and develop faster when acting together than either when acting alone, exhibiting a greater mixing depth [Aveiro and Hysell, 2010].

[6] The numerical simulations developed by Aveiro and Hysell [2010] incorporated a number of nonessential simplifications. Their model included time-invariant neutral atmosphere and background forcing. Also, the simulations were based on a single ion fluid model which did not take recombination into account. Here, we present the results of a multi-ion simulation which includes recombination, Coulomb collisions, a time-varying neutral atmosphere, and variable forcing, inferred from data from the C/NOFS satellite. The results are compared to incoherent scatter radar observations made by the ALTAIR radar in the Pacific sector.

[7] Key attributes of our simulation included (1) its sensitivity to the background circulation, (2) its sensitivity to event sequencing, and (3) its inclusion of parallel electric fields. The significance of the latter is not evaluated explicitly here and is left for a forthcoming analysis. However, it is worth mentioning that parallel ambipolar electric fields essentially changes the nature of the perpendicular fields, affecting convection and circulation [see, e.g., Zalesak et al., 1990; Oppenheim and Dimant, 2006]. The main issue we address below is the degree to which it is possible to predict, quantitatively, the behavior of ESF given an appropriate specification of the initial conditions and the forcing.

\section{Radar Observations}

[8] We begin with a presentation of the radar observations that will ultimately be used to motivate and validate the numerical simulations. The ARPA Long-Range Tracking and Instrumentation Radar (ALTAIR) is a VHF/UHF radar located at Kwajalein Atoll $\left(8.8^{\circ} \mathrm{N}, 167.5^{\circ} \mathrm{E}\right)$ [Tsunoda et al., 1979]. Figure 1 displays ALTAIR UHF (422 MHz) data for the night of 29 April 2009. ALTAIR observations made perpendicular to the geomagnetic field at $F$ region heights represent a combination of coherent and incoherent scatter, whereas off-perpendicular scans reflect only incoherent scatter. Some coherent scatter can be informative in data sets like these because it highlights fine structure that might otherwise be difficult to perceive.

[9] Figure 1 (top) shows an ALTAIR scan made pointing perpendicular to the geomagnetic field around 09:45 UT ( $\mathrm{LT} \approx \mathrm{UT}+11 \mathrm{~h}$ in the radar longitudinal sector). By this time, irregularities had formed at the base of the $F$ region as bottom-type layers. These were patchy and spaced by about $35 \mathrm{~km}$. They are common precursors of fully developed ESF, whereas bottom-type layers tend to be more continuous (beam filling) or absent on nights when topside ESF does not develop [Hysell et al., 2004].

[10] ALTAIR scans made pointing off perpendicular to the geomagnetic field at 10:30 UT and 11:26 UT are shown in Figures 1 (middle) and 1 (bottom), respectively. By about 10:30 UT, the irregularities were still confined to the base of the $F$ region, but the predominant horizontal scale had increased to 100-200 km. Between about 10:30 and 11:26 UT, the bottomside depletions underwent strong vertical development. Depleted channels penetrated to the topside while retaining their 100-200 km horizontal spacing. The depletions had characteristic tilts and showed evidence of bifurcation and secondary instabilities. Wedges of enhanced plasma also appeared to be driven downward in between ascending depletions.

\section{Satellite Observations}

[11] To drive simulations of the 29 April 2009 ESF event, in situ electric field measurements from the Ion Velocity Meter (IVM) and Vector Electric Field Instrument (VEFI) on board the Communications/Navigation Outage Forecasting System (C/NOFS) satellite were used. This satellite was launched in April 2008, to investigate and forecast ambient plasma density and irregularities in the equatorial $F$ region ionosphere [de La Beaujardiere, 2004]. IVM is composed of an ion drift meter and an ion-retarding potential analyzer that together provide measurements of the ion drift vector, the ion temperature, and the major ion composition. The VEFI instrument consists of an electric field meter, a fluxgate magnetometer, an optical lightning detector, and a fixed bias Langmuir probe. Among its products are measurements of DC, quasi-DC, and AC electric fields.

[12] Figure 2 shows the zonal electric field (based on IVM ion drift and VEFI magnetometer measurements) and (IVM) ion density measurements with the respective orbital information tabulated below (geographic and magnetic coordinates, altitude, and universal and solar local times). The results correspond to C/NOFS passes during the postsunset period on 29 April 2009, centered at the Kwajalein longitudinal sector. For comparison, satellite passes at $\sim 09: 03$ UT (Figure 2a), $\sim 10: 47$ UT (Figure $2 b$ ) and $\sim 12: 31$ UT (Figure 2c) are shown.

[13] During the first C/NOFS pass at about 09:03 UT, the zonal electric field was essentially zero, and no density or electric field perturbations were detected in the altitude range between 495 and $520 \mathrm{~km}$. By the time of the next pass at about 10:47 UT, the zonal electric field had increased to $0.5-0.75 \mathrm{mV} \mathrm{m}^{-1}$, and small electric field irregularities were emerging between 435 and $450 \mathrm{~km}$ altitude. That the background plasma density increased as the satellite altitude decreased suggests that it was flying in the topside, which is consistent with the evidence of Figure 1.

[14] By the time the satellite passed through the Kwajalein sector again at about 12:30 UT, the electric field irregularities had become large (up to about $4 \mathrm{mV} \mathrm{m}^{-1}$ ). Modest plasma density irregularities were also present at altitudes near $400 \mathrm{~km}$. In view of Figure 1, however, the satellite most likely flew just above the most intense plasma density irregularities.

\section{Numerical Simulations}

[15] Below, we describe a numerical scheme for the simulation of the plasma density irregularities in the equatorial spread $F$ event. The 3-D simulation updates the plasma density and electrostatic potential in time assuming quasineutrality and momentum balance. Inertia is neglected in the 

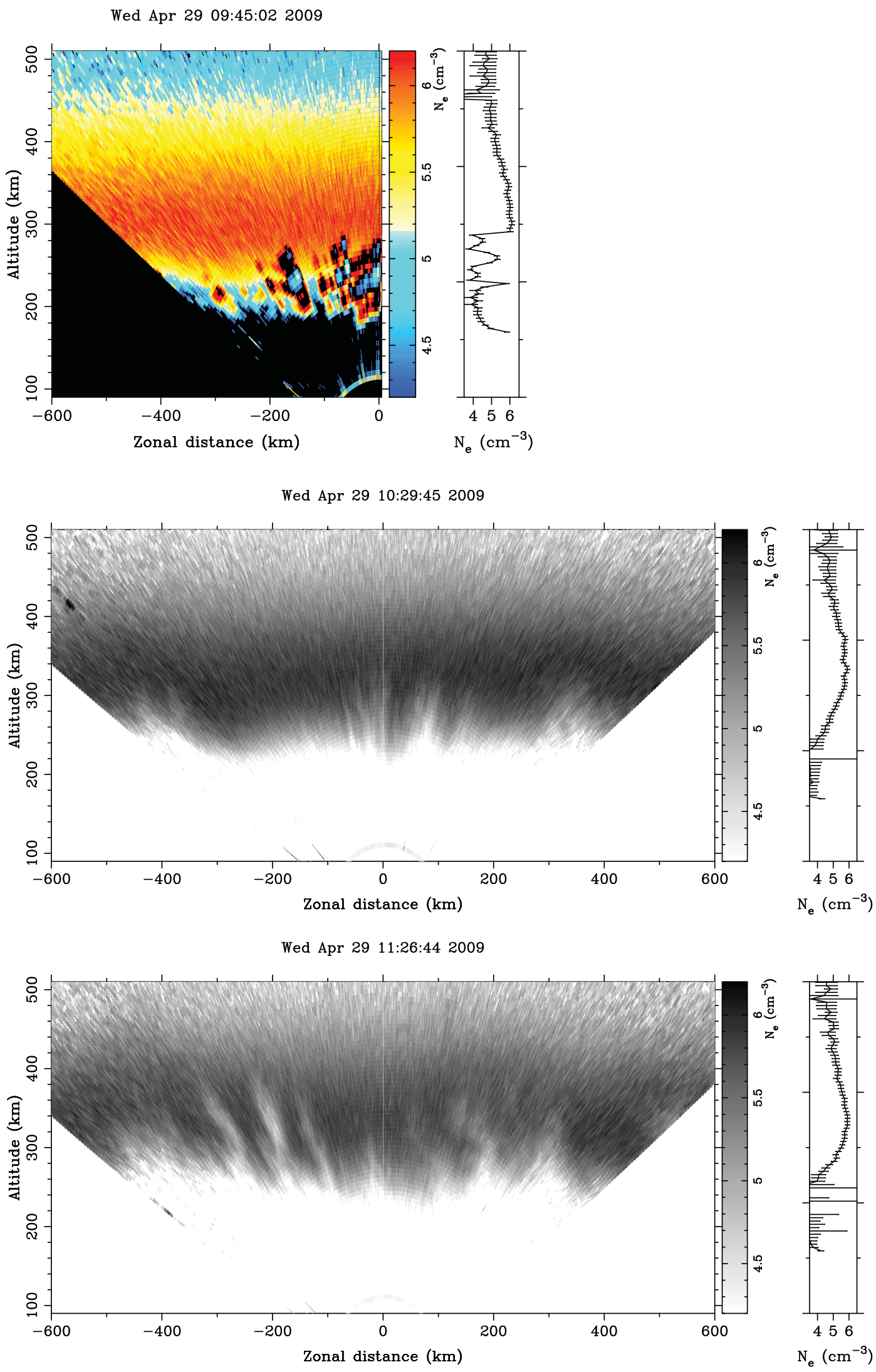

Figure 1. (left) ALTAIR radar scans for 29 April 2009, showing backscatter power, range corrected and scaled to electron density on a logarithmic scale: (top) perpendicular scan at 09:45 UT and off-perpendicular scans at (middle) 10:30 UT and (bottom) 11:26 UT. (right) Vertical electron density profiles. Note that $\mathrm{LT} \approx \mathrm{UT}+11 \mathrm{~h}$ 

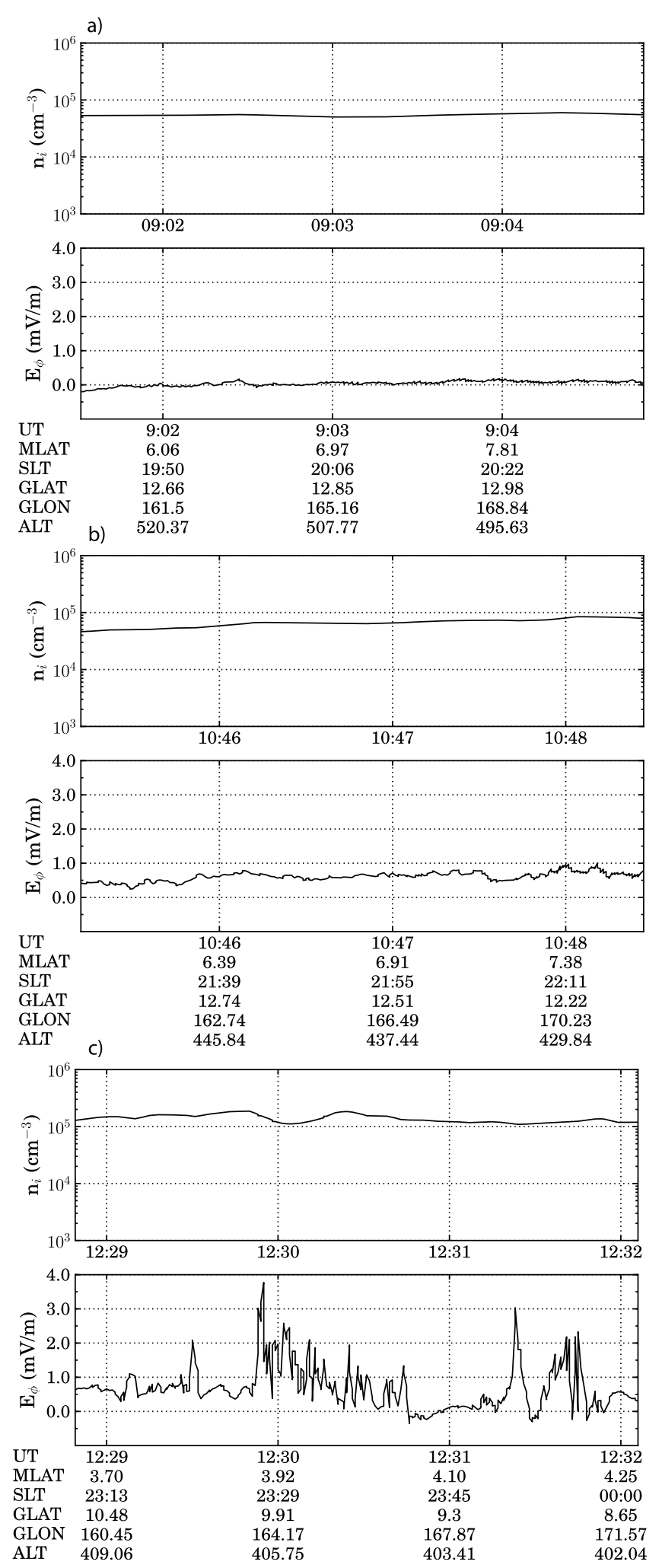

Figure 2. Ion density $\left(\mathrm{cm}^{-3}\right)$ and zonal electric field $\left(\mathrm{mV} \mathrm{m}^{-1}\right)$ at three different passes through the Kwajalein sector: (a) 09:03 UT, (b) 10:47 UT, and (c) 12:30 UT. The tabulated entries are universal time (UT), magnetic latitude (MLAT), solar local time (SLT), geographic latitude (GLAT), longitude (GLON), and altitude (ALT) in km. present incarnation. The characteristics of the neutral atmosphere are imported from empirical models. The background electric field is specified and controls the forcing. Simulations are performed with realistic background conditions including bottomside plasma shear flow and the attendant vertical current.

[16] Rather than assuming equipotential magnetic field lines, the simulation solves the potential equation in three dimensions, allowing us to keep track of the distribution of parallel currents forced by the dynamos and their closure. This also accommodates the possible effects of drift waves and related instabilities that may play secondary roles in ESF [Huba and Ossakow, 1979; LaBelle et al., 1986; Hysell et al., 2002]. For instance, the full 3-D treatment of warm plasma shows that perturbations on the surface of ionospheric plasma clouds propagate and twist into a barber pole configuration [Drake and Huba, 1987; Drake et al., 1988; Zalesak et al., 1990]. As a consequence, the phase of the corrugations varies strongly along $\mathbf{B}$, the plasma cloud loses its flute-like characteristic (i.e., $\mathbf{k} \cdot \mathbf{B} \neq 0$ ), and the resulting finite $k_{\|}$has a dissipative influence on the instability. We do not know the extent to which similar phenomenon might affect the ESF development, but a study is underway. Finally, it is worth mentioning that magnetic field lines cease to be equipotentials near the bottom of the $E$ region, decoupling the plasma and decreasing the conductive loading on the dynamo, which may influence the overall circulation.

\subsection{Conservation Laws}

[17] Neglecting stress, heat flow, and inertia, the fluid continuity equation and momentum equations for electrons and ions are given as

$$
\begin{gathered}
\frac{\partial n_{\alpha}}{\partial t}+\nabla \cdot\left(n_{\alpha} v_{\alpha}\right)=P_{\alpha}-L_{\alpha}, \\
0=-\nabla p_{\alpha}+n_{\alpha} q_{\alpha}\left(\mathbf{E}+\mathbf{v}_{\alpha} \times \mathbf{B}\right) \\
+m_{\alpha} n_{\alpha}\left[\mathbf{g}-\nu_{\alpha n}\left(\mathbf{v}_{\alpha}-\mathbf{U}\right)-\sum_{\beta}^{\alpha \neq \beta} \nu_{\alpha \beta}\left(\mathbf{v}_{\alpha}-\mathbf{v}_{\beta}\right)\right],
\end{gathered}
$$

where $p_{a}=n_{\alpha} k_{B} T_{\alpha}$ is the pressure. The variables $n_{\alpha}, q_{\alpha}, m_{\alpha}$, $T_{\alpha}, P_{\alpha}, L_{\alpha}$, and $\mathbf{v}_{\alpha}$ are number density, electric charge, mass, temperature, production and loss rates, and velocity of the species $\alpha$, respectively. The loss term includes charge exchange and dissociative recombination for a given species. Nighttime photoionization is neglected, but production due to charge exchange is considered. Here, $\beta$ represents the other ionized bodies that exchange momentum or energy with $\alpha$. The terms $\nu_{\alpha \beta}$ and $\nu_{\alpha n}$ are the collision frequencies with charged particles and neutrals, respectively. The terms $\mathbf{E}, \mathbf{B}, \mathbf{U}, \mathbf{g}$, and $k_{B}$ represent the electric field, magnetic field, neutral wind, gravity, and the Boltzmann constant, respectively. Equation (1) is not in conservation form, since it includes chemical processes. However, it can be converted to conservative form through variable transformation. Two simplifications are applied to equations (1) and (2): (1) the neglect of diagmagnetic drifts $\left(\nabla n_{\alpha} \times \mathbf{B}\right)$, since their associated flux divergence is very small when compared to the other terms, and (2) Coulomb collisions (the last term on the right-hand side of equation (2)) are treated implicitly and only included in the parallel direction. 


\subsection{Numerical Scheme}

[18] The simulation algorithm performs two computations. First, the self-consistent electric field is found using a 3-D potential solver based on quasi-neutrality, which can be expressed as

$$
\nabla \cdot[\hat{\Sigma} \cdot \nabla \Phi]=\nabla \cdot\left[\hat{\Sigma} \cdot\left(\mathbf{E}_{o}+\mathbf{U} \times \mathbf{B}\right)+\hat{D} \cdot \nabla n+\hat{\Xi} \cdot \mathbf{g}\right],
$$

where $\mathbf{E}_{o}$ is the background electric field, $\hat{\Sigma}$ is the conductivity tensor, $\hat{D}$ includes the diffusivity tensor, $\hat{\Xi} \cdot \mathbf{g}$ is gravitydriven current density, and $\Phi$ is the electrostatic potential that must arise to preserve a solenoidal current density. Explicit definitions of the tensors are given by Shume et al. [2005]. Note that the resulting electric field is the combination of the background and electrostatic fields (i.e., $\mathbf{E}=$ $\left.\mathbf{E}_{o}-\nabla \Phi\right)$. Equation (3) involves a partial differential equation for the potential in three dimensions. It is solved using the BiConjugate Gradient Stabilized (BiCGStab) method [e.g., van der Vorst, 1992] using the algorithms described by Saad [1990].

[19] Second, we solve a discretized version of equation (1) for each ion species using a monotone upwind scheme for conservation laws (MUSCL) (a pedagogical review is given by Trac and Pen [2003]) directly applicable to the ion continuity problem (once the recombination term has been absorbed into a redefinition of $n_{\alpha}$ so that equation (1) becomes a conservation equation, as necessary). The characteristic of the neutral atmosphere (densities, temperature, and wind velocity) are updated in time on the basis of inputs from climatological models. The background electric field is specified and partly controls the forcing. The ion composition includes $\mathrm{O}^{+}, \mathrm{NO}^{+}$, and $\mathrm{O}_{2}^{+}$. The model was constructed using tilted magnetic dipole coordinates $(p, q, \phi)$, where the tilt is matched to the magnetic declination in the longitude of interest. In our terminology, $p$ represents the McIlwain parameter $(L), q$ is the magnetic colatitude, and $\phi$ is longitude [see, e.g., Hysell et al., 2004].

\subsection{Empirical Model Drivers}

[20] To initialize the model runs, we derive plasma number densities from the Parametrized Ionospheric Model (PIM), a parametrization of the output of several regional theoretical model outputs generated for different climatological conditions and tuned somewhat to agree with data from Jicamarca and elsewhere [Daniell et al., 1995]. PIM reproduces electron density profiles from Kwajalein and Jicamarca fairly closely if scaled slightly to account for dayto-day variability [Aveiro and Hysell, 2010]. The background number density profiles used for initial conditions are intended to reproduce measurements from ALTAIR, including the $F$ region peak height and peak density, the bottomside density scale height, and the density in the valley region.

[21] Plasma mobilities are calculated using neutral composition and temperature estimates from the Mass Spectrometer and Incoherent Scatter (NRL-MSISE00) model [Picone et al., 2002] and ionospheric composition estimates from the International Reference Ionosphere (IRI-2007) model [Bilitza and Reinisch, 2008]. We take the ionosphere and neutral atmosphere to be in thermodynamic equilibrium after sunset $\left(T_{n}=T_{e}=T_{i}\right)$. Expressions for the ion-neutral and electron-neutral collision frequencies used to compute conductivities can be taken from Richmond [1972]. Expressions for the Pedersen, Hall, and direct mobilities and diffusivities themselves are given, e.g., by Kelley [2009].

[22] For our runs, the zonal neutral winds are obtained from the Horizontal Wind Model (HWM-07) [Drob et al., 2008]. As with PIM, these may be tuned slightly to account for day-to-day variability. The model is sensitive to the wind speeds throughout the thermosphere, since the growth rate of the collisional shear instability depends on the counter streaming flow of the neutrals and plasma. Meridional and vertical winds were neglected in the simulations presented here.

\subsection{Simulation Setup}

[23] Our simulation was cast on a rectangular grid $139 \times$ $133 \times 189$ points wide in $(p, q, \phi)$ space constructed using a tilted magnetic dipole coordinate system. A cut through the equatorial plane spans altitudes between 90 and $510 \mathrm{~km}$ and longitudes of $\pm 6^{\circ}$. The flux tubes covered by the parallel coordinate all reach to the lower $E$ region. The runs shown in this section are centered on the dip equator near Kwajalein $\left(5.5^{\circ} \mathrm{N}\right.$ latitude, $166.5^{\circ} \mathrm{E}$ longitude, $7.4^{\circ}$ declination) with background conditions modeled for 29 April 2009. To seed the simulation run, we added independent Gaussian white noise to the initial number density with a $20 \%$ relative amplitude. The local time dependence of the background zonal electric field $\left(E_{\phi}\right)$ was estimated using measurements from IVM and VEFI on board the C/NOFS satellite. $E_{\phi}$ was taken to be constant in the $F$ region but its amplitude is reduced below the valley to avoid the growth of electrojet gradient drift instabilities. Based on the C/NOFS measurements $E_{\phi}$ was modeled as $0 \mathrm{mV} \mathrm{m}^{-1}$ before 21:00 LT, and $0.75 \mathrm{mV} \mathrm{m}^{-1}$ after 22:00 LT with a gradual transition using a hyperbolic tangent function in between. For example, $E_{\phi}=34 \mu \mathrm{V} \mathrm{m}{ }^{-1}$ on the western boundary and $0.75 \mathrm{mV} \mathrm{m}^{-1}$ on the eastern boundary of the simulation by 10:40 UT, since those locations represent 21:22 LT and 22:10 LT, respectively.

\section{Simulation Results}

[24] The initial conditions of our simulation at $t_{0}=$ 9:30 UT $(\approx 20: 30$ LT for the Kwajalein sector) are shown in Figure 3. Figure 3 (top) shows the background plasma density. The initial conditions are consistent with ALTAIR radar observations for 29 April 2009, at that time. Figure 3 (middle) shows the perpendicular current density with the magnitude and direction indicated by the color disc in Figure 3 (bottom). There are two major components to the current density that are combined in this depiction. First, a zonally eastward current driven by gravity exists throughout the region, showing a maximum at the $F$ peak near $330 \mathrm{~km}$ altitude. In addition, a zonally eastward $F$ region wind drives a vertical upward current in the region above about $200 \mathrm{~km}$. The empirically modeled wind is almost constant with altitude, and thus the vertical current scales like the background ion density, showing a peak near $330 \mathrm{~km}$ and a strong vertical gradient in the bottomside between $280 \mathrm{~km}$ and $240 \mathrm{~km}$ altitude. Below $150 \mathrm{~km}$, the current is influenced by both the local zonal winds and the enhancement of the zonal current in the electrojet. 


\section{Electron Density}

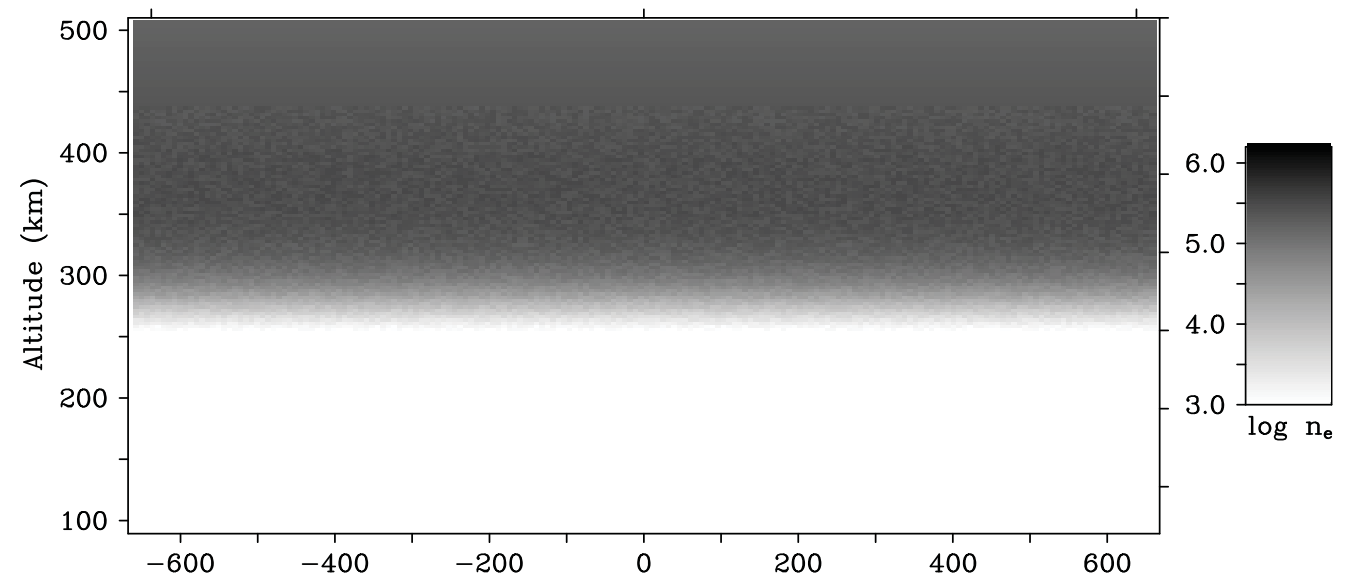

Transverse Currents

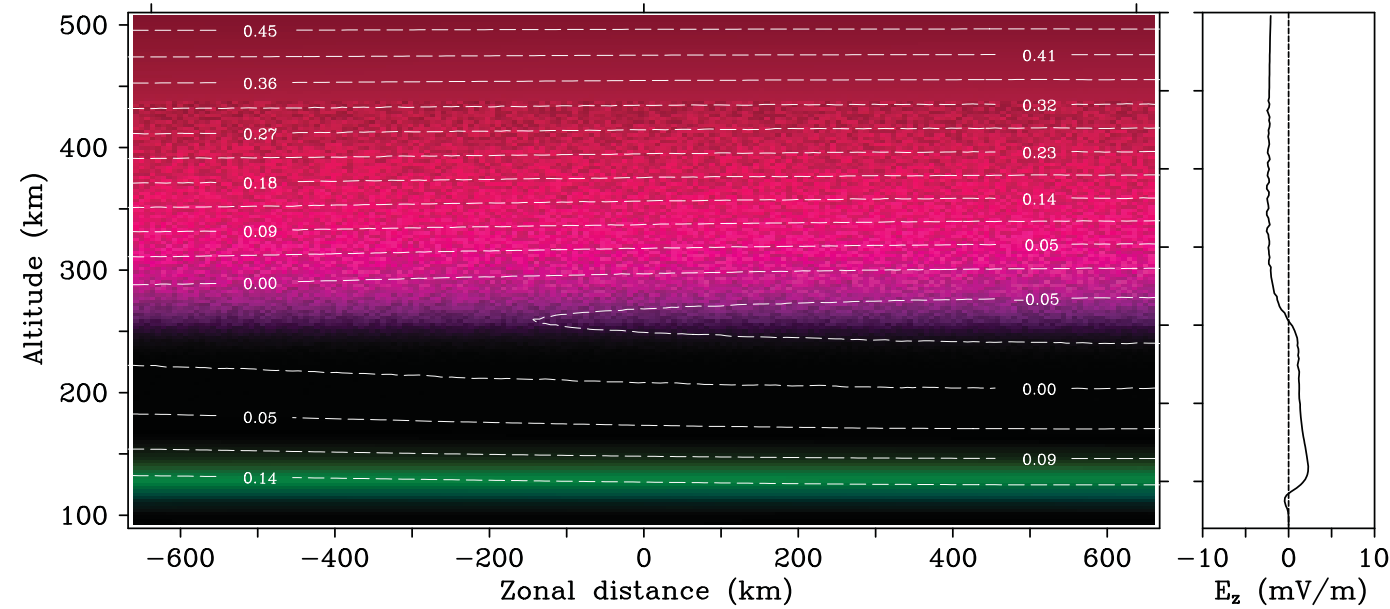

Meridional Currents

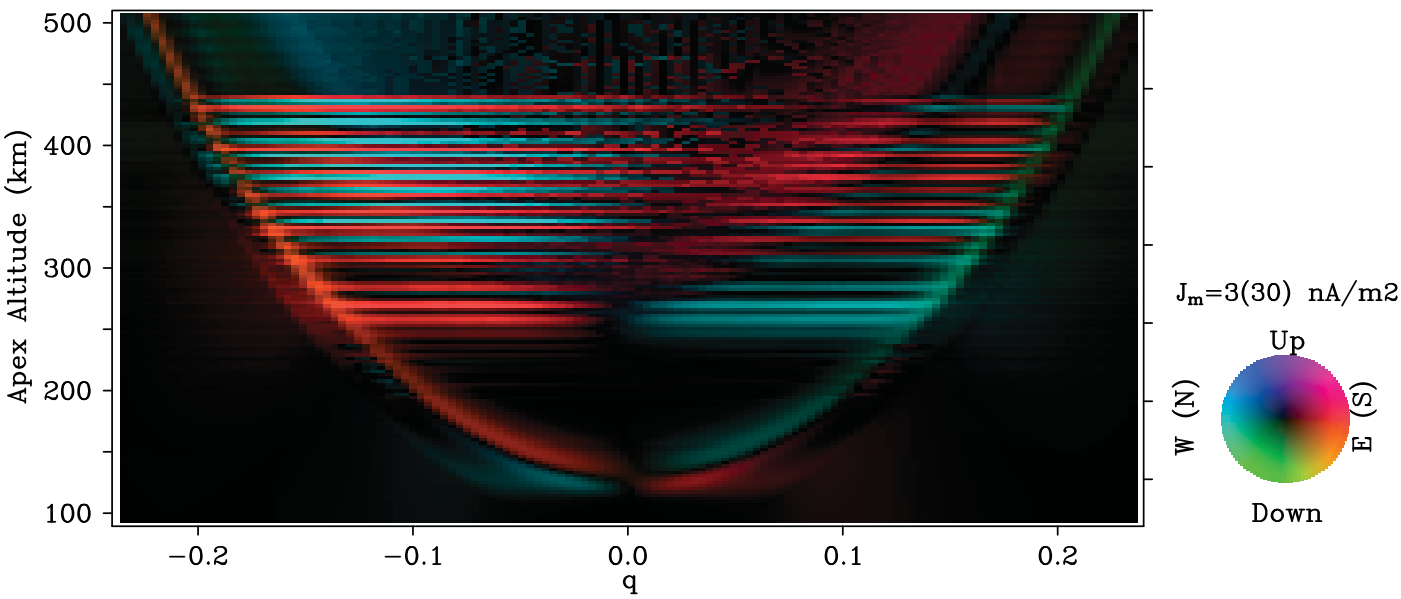

Figure 3. Initial conditions for ESF model run (i.e., at $t_{0}=09: 30 \mathrm{UT}$ ). (top) Plasma number density. (middle) Transverse current density in the equatorial plane with (left) equipotential contours (in $\mathrm{kV}$ ) superimposed in white and (right) vertical electric field. (bottom) Current density in the meridional plane. The current densities are vector quantities with magnitudes and directions indicated by the color wheel in Figure 3 (bottom right). Information outside and inside parentheses characterizes currents in the equatorial (Figure 3, middle) and meridional plane (Figure 3, bottom), respectively. The maximum scale is $3 \mathrm{nA} \mathrm{m}^{-2}$ in the equatorial plane and $30 \mathrm{nA} \mathrm{m}^{-2}$ in the meridional plane. Note that diamagnetic currents are not represented in this diagnostic figure. 

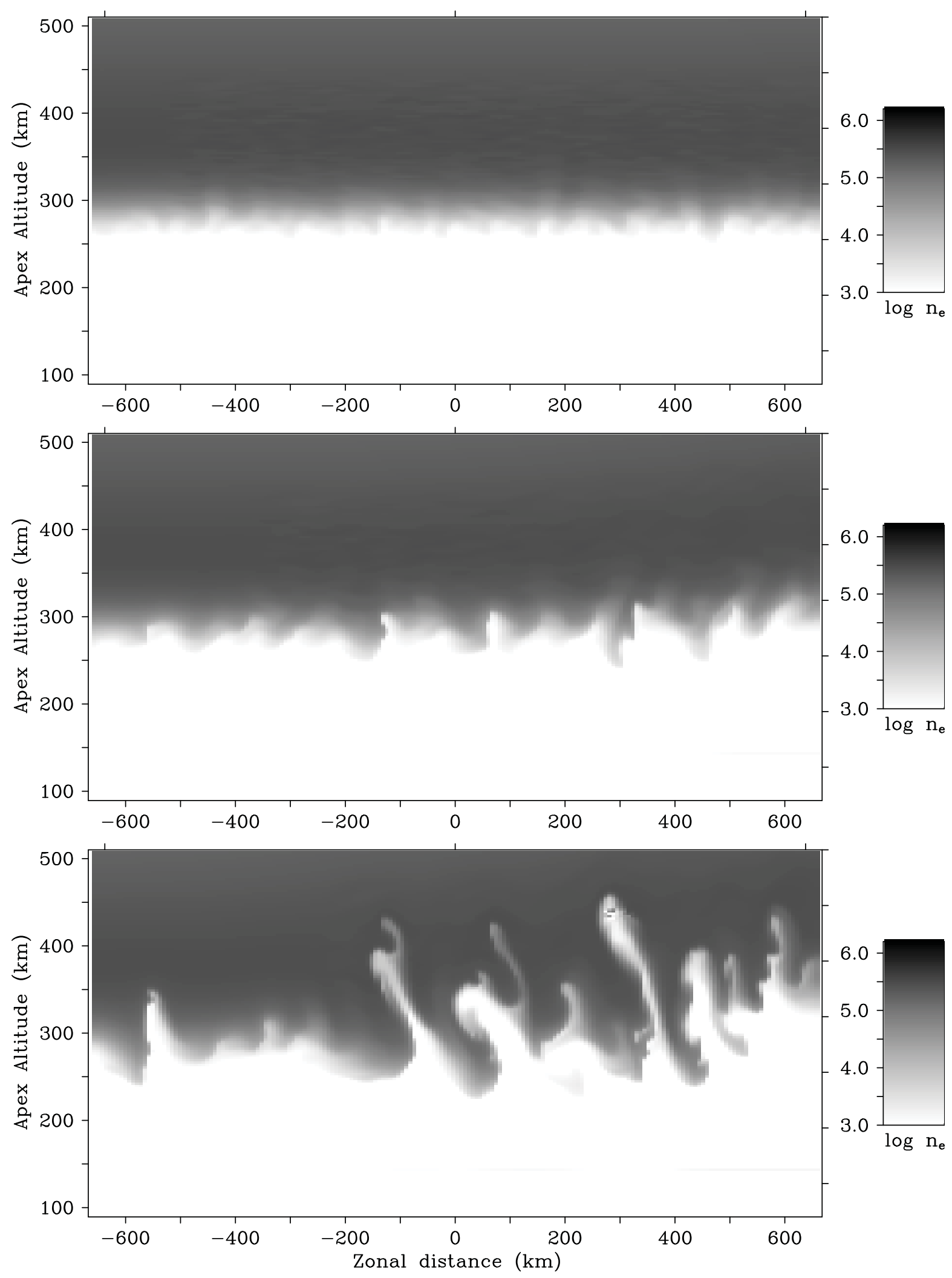

Figure 4. Simulated plasma densities in a cut through the Kwajalein latitudinal plane. Results are shown for simulation times of (top) 00:35, (middle) 01:10, and (bottom) 01:43, respectively, after a start time $t_{0}=09: 30 \mathrm{UT}(\mathrm{LT}=\mathrm{UT}+11 \mathrm{~h}$ at the horizontal center of the simulation). 


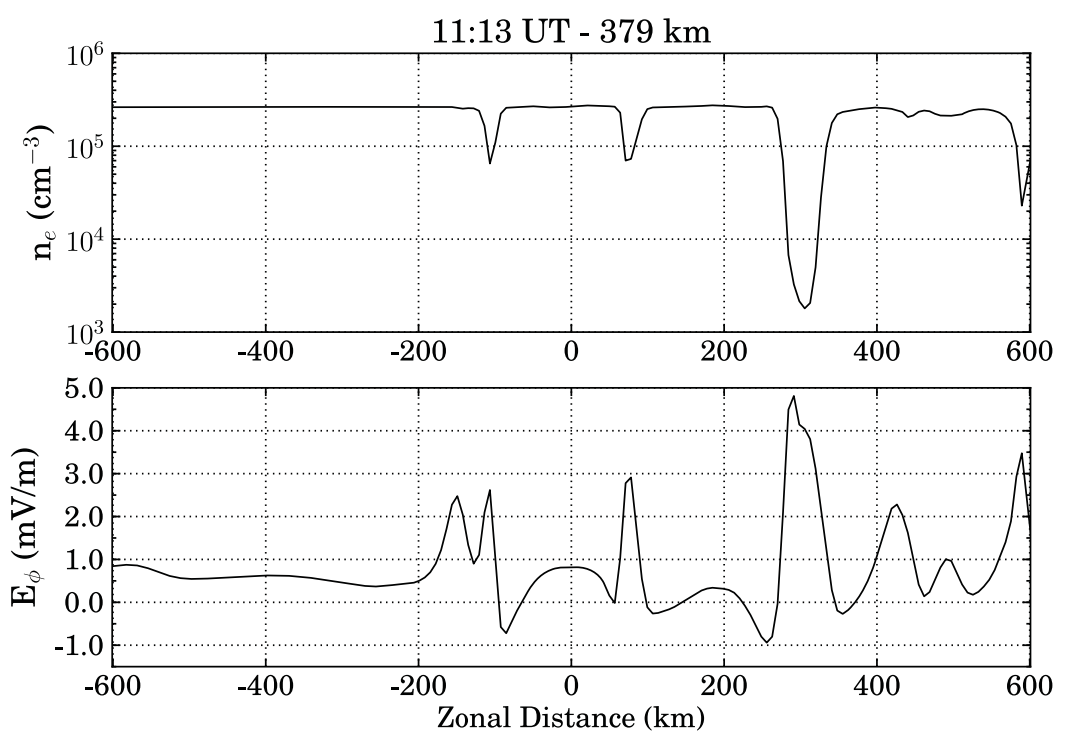

Figure 5. (top) Electron density $\left(\mathrm{cm}^{-3}\right)$ and (bottom) zonal electric field $\left(\mathrm{mV} \mathrm{m}^{-1}\right)$ at $11: 13 \mathrm{UT}$ obtained from a longitudinal cut through the simulation at $379 \mathrm{~km}$ altitude over the Kwajalein sector ( $\sim 400 \mathrm{~km}$ apex altitude).

[25] The divergence in the vertical current density is supported by a vertical electric field shown in Figure 3 (middle right). Above the bottomside, the field is directed downward, consistent with an eastward $\mathbf{E} \times \mathbf{B}$ drift in the same direction as the dynamo wind. Across the bottomside, the vertical electric field changes direction, producing a sheared zonal $\mathbf{E} \times \mathbf{B}$ drift, which provides free energy for the CSI below the shear node due to the differential velocity between plasma and neutrals.

[26] Figure 3 (bottom) shows equatorward currents in both hemispheres on field lines that thread the bottomside in the altitude range from $250 \mathrm{~km}$ to $280 \mathrm{~km}$. These currents feed the upward current driven by the $F$ region zonal wind seen in Figure 3 (middle). Above the bottomside, the meridional current is distributed across all the field lines and is poleward in both hemispheres, serving to close the current loop driven by the $F$ region dynamo.

[27] Figure 4 shows the results for times matching, roughly, the three ALTAIR scans and C/NOFS passes. Note that the vertical axes indicate the apex altitude, i.e., the altitude mapped to the magnetic equator. For example, the magnetic field line that crosses over Kwajalein at $380 \mathrm{~km}$ altitude maps to $400 \mathrm{~km}$ altitude at the magnetic equator.

[28] Figure 4 (top) depicts the evolution of ionospheric irregularities in the absence of a background electric field due entirely to the collisional shear instability. The spatial scales of the irregularities forming at the base of the bottomside range between 30 and $50 \mathrm{~km}$. These irregularities cannot undergo vertical development and remain confined to altitudes where the plasma flow is retrograde.

[29] By the time of Figure 4 (middle), the transient phase of the CSI was ending, and the asymptotic phase was underway. The $30-50 \mathrm{~km}$ waves at the base of the bottomside have coalesced into large-scale waves with scale sizes between 100 and $200 \mathrm{~km}$. Under the action of an increasing background zonal electric field, the bottomside irregularities begin to exhibit vertical development, more so on the eastern side of the simulation volume, which has experienced an eastward background electric field for more time.

[30] In Figure 4 (bottom), under the influence of a significant background electric field, the irregularities penetrate to the topside where gravity-driven currents can contribute further to growth. Some of the original intermediate-scale structure from Figure 4 (top) in the simulation survive, contributing to the overall fine structure. Bifurcation is evident, as well as secondary instabilities growing mainly on the western walls of the primaries. Some descending, wedgeshaped plasma enhancements accompany the ascending depletions. The characteristic westward tilt of the irregularities is associated with the combination of vertical and zonal currents and the combined CSI and GRT instabilities. As the simulation was initialized with white noise, and as GRT is a universal instability (nearly scale size independent), the scale sizes of the irregularities evident here are a consequence of CSI.

[31] Had a satellite passed through the simulated ionosphere at $379 \mathrm{~km}$ altitude over the Kwajalein sector ( $\sim 400 \mathrm{~km}$ apex altitude) at about 11:13 UT, the electron density and electric field perturbations would have appeared as shown in Figure 5. Plasma depletions are present preferentially in the eastern half of the simulation grid. The western walls of the irregularities have steeper density gradients than the eastern walls. Perturbations in the zonal electric fields of the order $3-5 \mathrm{mV} \mathrm{m}^{-1}$ are present, collocated with large and small plasma depletions alike. Since the pass is a few kilometers above the top of most of the plasma depletions, relative perturbations in the density are small in all but one case, but the fringing fields penetrate to higher altitudes and are detectable in all cases.

\section{Discussions}

[32] In simulation, the combination of CSI and GRT produced growing waveforms with characteristics that matched 
the observations made by ALTAIR radar. The dominant plasma irregularities with (initially) decakilometric-scale took approximately $100 \mathrm{~min}$ to evolve from the bottomside up to the $F$ peak. By the time they reached the $F$ peak, they have coalesced into waveforms with $100+\mathrm{km}$ scale sizes. This behavior was observed in ALTAIR scans and simulation results.

[33] The transient response of CSI explained $\sim 30-50 \mathrm{~km}$ wave observations, a feature commonly observed by backscatter scatter radars in the form of patchy bottom-type ESF. These intermediate-scale irregularities were observed initially in both the simulations and the radar observations but did not reappear after they coalesced into emergent $100+\mathrm{km}$ scale irregularities. This result agrees with observations made at other longitudinal sectors, e.g., at Jicamarca [Hysell, 2000].

[34] During the asymptotic growth phase, when intermediate-scale depletions dominated the scenario, some of the irregularities remained confined to the bottomside, while others experienced more vertical development. Had these irregularities been observed with coherent scatter, e.g., by the Jicamarca radar, they would have been labeled bottomside ESF. The irregularities in the simulation in Figure 4 (middle) and radar data in Figure 1 (top) are comparable in terms of scale sizes and altitude spans.

[35] The last stage of development of ESF occurred when the vertical extension of the plasma depletion was large enough that the irregularities crossed through the peak into the topside. Similar features are observed in both the simulation (Figure 4, bottom) and the radar data (Figure 1, bottom). Depletions that reached the $F$ peak were spaced by hundreds of $\mathrm{km}$ and displayed tilts close to $30^{\circ}$ westward from zenith.

[36] Measurements made by IVM showed that electric field perturbations of the order of a few $\mathrm{mV} \mathrm{m}^{-1}$ were sometimes observed even when depletions in the densities were not detected. Similar features were present in the simulation and seemed to be a natural characteristic of the ESF morphology. The simultaneous measurements of small relative perturbations in the density and large zonal electric field perturbations seems to be a consequence of a satellite pass near the top of a plasma depletion. Since the depletion itself was not intercepted, it was not observed, but the fringing fields penetrated to higher altitudes and were detected.

[37] Zonal neutral winds in the bottomside and valley regions exerted control over initial irregularity formation. This sensitivity to the winds was expected, since the growth rate of the collisional shear instability depends on the counter streaming flow of the neutrals and plasma [Hysell et al., 2006]. As wind measurements in the lower thermosphere and mesosphere show considerable variability [Larsen, 2002], sensitivity tests to winds at those altitudes need to be performed to evaluate their effects on ESF initiation.

\section{Summary}

[38] The combination of generalized Rayleigh-Taylor and collisional shear instability produced growing waveforms with characteristics matching satellite and radar observations in terms of their gross morphology and rates of development. Growing intermediate- $(\sim 30 \mathrm{~km})$ and large-scale $(100+\mathrm{km})$ waves resulted from the nonlocal and nonnormal characteristics of the system and were not imposed a priori. The transient response of CSI explained rapid intermediatescale wave emergence at the base of the $F$ region, whereas the steady state behavior seemed to account for the largescale waves that ultimately dominated. The three different types of ESF (bottom-type, bottomside, and topside) appeared to be a result of the time sequencing of the transient and steady state response of CSI in relation to the zonal electric field.

[39] A major objective of this research was to simulate the electrodynamics of ionospheric plasma irregularities, including those in ESF, in the direction parallel to the geomagnetic field. The results of ESF simulation presented here suggests that a full 3-D treatment is necessary in order to track the distribution of parallel currents and the parallel closure of currents forced by dynamos. Parallel currents are found to have effects on the overall circulation and contribute to ESF morphology. Studies are underway to quantify the effects and mechanisms at work.

[40] Acknowledgments. This work was supported by award FA955009-1-0337 from the Air Force Office of Sponsored Research to Cornell University. The CINDI project is supported at the University of Texas at Dallas by NASA grant NNX10AM94G. Special thanks go to Dale Sponseller and the rest of the Kwajalein Range Systems crew at the Kwajalein Missile Range for their support during the 2009 C/NOFS campaign.

[41] Robert Lysak thanks the reviewers for their assistance in evaluating this paper.

\section{References}

Aveiro, H. C., and D. L. Hysell (2010), Three-dimensional numerical simulation of equatorial $F$ region plasma irregularities with bottomside shear flow, J. Geophys. Res., 115, A11321, doi:10.1029/2010JA015602.

Bilitza, D., and B. W. Reinisch (2008), International Reference Ionosphere 2007: Improvements and new parameters, Adv. Space Res., 42, 599-609.

Daniell, R. E., Jr., L. D. Brown, D. N. Anderson, M. W. Fox, P. H. Doherty,

D. T. Decker, J. J. Sojka, and R. W. Schunk (1995), Parameterized ionospheric model: A global ionospheric parameterization based on first principles models, Radio Sci., 30(5), 1499-1510, doi:10.1029/95RS01826.

de La Beaujardiere, O. (2004), C/NOFS: A mission to forecast scintillations, J. Atmos. Sol. Terr. Phys., 66, 1573-1591, doi:10.1016/j.jastp.2004.07.030.

Drake, J. F., and J. D. Huba (1987), Dynamics of three-dimensional ionospheric plasma clouds, Phys. Rev. Lett., 58, 3, 278-281.

Drake, J. F., M. Mulbrandon, and J. D. Huba (1988), Three-dimensional equilibrium and stability of ionospheric plasma clouds, Phys. Fluids, $31,3412-3424$.

Drob, D. P., et al. (2008), An empirical model of the Earth's horizontal wind fields: HWM07, J. Geophys. Res., 113, A12304, doi:10.1029/ 2008JA013668

Haerendel, G., J. V. Eccles, and S. Çakir (1992), Theory for modeling the equatorial evening ionosphere and the origin of the shear in the horizontal plasma flow, J. Geophys. Res., 97(A2), 1209-1223, doi:10.1029/ 91JA02226.

Huba, J. D., and S. L. Ossakow (1979), On the generation of 3-m irregularities during equatorial spread $F$ by low-frequency drift waves, J. Geophys. Res., 84(A11), 6697-6700, doi:10.1029/JA084iA11p06697.

Huba, J. D., G. Joyce, and J. Krall (2008), Three-dimensional equatorial spread F modeling, Geophys. Res. Lett., 35, L10102, doi:10.1029/ 2008GL033509.

Hysell, D. L. (2000), An overview and synthesis of plasma irregularities in equatorial spread F, J. Atmos. Sol. Terr. Phys., 62, 1037-1056, doi:10.1016/S1364-6826(00)00095-X.

Hysell, D. L., and E. Kudeki (2004), Collisional shear instability in the equatorial $F$ region ionosphere, J. Geophys. Res., 109, A11301, doi:10.1029/ 2004JA010636.

Hysell, D. L., M. Yamamoto, and S. Fukao (2002), Imaging radar observations and theory of type I and type II quasi-periodic echoes, J. Geophys. Res., 107(A11), 1360, doi:10.1029/2002JA009292.

Hysell, D. L., J. Chun, and J. L. Chau (2004), Bottom-type scattering layers and equatorial spread $F$, Ann. Geophys., 22, 4061-4069.

Hysell, D. L., M. F. Larsen, C. M. Swenson, A. Barjatya, T. F. Wheeler, T. W. Bullett, R. F. W. M. F. Sarango, J. L. Chau, and D. Sponseller (2006), Rocket and radar investigation of background electrodynamics 
and bottom-type scattering layers at the onset of equatorial spread $F$, Ann. Geophys., 24, 1387-1400, doi:10.5194/angeo-24-1387-2006.

Kelley, M. C. (2009), The Earth's Ionosphere: Plasma Physics and Electrodynamics, Academic, Amsterdam.

Keskinen, M. J. (2010), Equatorial ionospheric bubble precursor, Geophys. Res. Lett., 37, L09106, doi:10.1029/2010GL042963.

Kudeki, E., A. Akgiray, M. A. Milla, J. L. Chau, and D. L. Hysell (2007), Equatorial spread-F initiation: Post-sunset vortex, thermospheric winds, gravity waves, J. Atmos. Sol. Terr. Phys., 69, 2416-2427.

LaBelle, J., M. C. Kelley, and C. E. Seyler (1986), An analysis of the role of drift waves in equatorial spread $F, J$. Geophys. Res., 91(A5), 5513-5525, doi:10.1029/JA091iA05p05513.

Larsen, M. F. (2002), Winds and shears in the mesosphere and lower thermosphere: Results from four decades of chemical release wind measurements, J. Geophys. Res., 107(A8), 1215, doi:10.1029/2001JA000218.

Oppenheim, M. M., and Y. Dimant (2006), Meteor induced ridge and trough formation and the structuring of the nighttime E-region ionosphere, Geophys. Res. Lett., 33, L24105, doi:10.1029/2006GL028267.

Picone, J. M., A. E. Hedin, D. P. Drob, and A. C. Aikin (2002), NRLMSISE-00 empirical model of the atmosphere: Statistical comparisons and scientific issues, J. Geophys. Res., 107(A12), 1468, doi:10.1029/ 2002JA009430.

Retterer, J. M. (2010), Forecasting low-latitude radio scintillation with 3-D ionospheric plume models: 1. Plume model, J. Geophys. Res., 115, A03306, doi:10.1029/2008JA013839.

Richmond, A. D. (1972), Numerical model of the equatorial electrojet, Tech. Rep. AFCRL-72-0668, ERP 421, Air Force Cambridge Res. Lab., Hanscom AFB, Bedford, Mass.

Saad, Y. (1990), SPARSKIT : A basic tool kit for sparse matrix computations, Tech. Rep. RIACS-90-20, Res. Inst. for Adv. Comput. Sci., NASA Ames Res. Cent., Moffett Field, Calif.
Shume, E. B., D. L. Hysell, and J. L. Chau (2005), Zonal wind velocity profiles in the equatorial electrojet derived from phase velocities of type II radar echoes, J. Geophys. Res., 110, A12308, doi:10.1029/2005JA011210. Sultan, P. J. (1996), Linear theory and modeling of the Rayleigh-Taylor instability leading to the occurrence of equatorial spread $F, J$. Geophys. Res., 101(A12), 26,875-26,891, doi:10.1029/96JA00682.

Trac, H., and U. L. Pen (2003), A primer on Eulerian computational fluid dynamics for astrophysicists, Astrophysics, 115, 303-321.

Tsunoda, R. T., M. J. Baron, J. Owen, and D. M. Towle (1979), Altair: An incoherent scatter radar for equatorial spread $F$ studies, Radio Sci., 14(6), 1111-1119, doi:10.1029/RS014i006p01111.

van der Vorst, H. (1992), Bi-CGSTAB: A fast and smoothly converging variant of $\mathrm{Bi}-\mathrm{CG}$ for the solution of nonsymmetric linear systems, SIAM J. Sci. Stat. Comput., 13, 631-644.

Woodman, R. F. (2009), Spread F-An old equatorial aeronomy problem finally resolved?, Ann. Geophys., 27, 1915-1934.

Zalesak, S. T., J. F. Drake, and J. D. Huba (1990), Three-dimensional simulation study of ionospheric plasma clouds, Geophys. Res. Lett., 17(10), 1597-1600, doi:10.1029/GL017i010p01597.

H. C. Aveiro and D. L. Hysell, Earth and Atmospheric Sciences, Cornell University, 2122 Snee Hall, Ithaca, NY 14853, USA. (hca24@cornell.edu)

R. G. Caton, Space Vehicles Directorate, Air Force Research Laboratory, 3550 Aberdeen Ave., Kirtland AFB, NM 87117, USA.

K. M. Groves, Space Vehicles Directorate, Air Force Research Laboratory, 29 Randolph Rd., Hanscom AFB, MA 01731-3010, USA.

R. A. Heelis and R. Stoneback, Hanson Center for Space Sciences, University of Texas at Dallas, 800 W. Campbell Rd., WT15, Richardson, TX 75080, USA.

J. Klenzing and R. F. Pfaff, NASA Goddard Space Flight Center, Code 674, Greenbelt, MD 20771, USA. 